\title{
Molecular Mechanisms and Differences in Lynch Syndrome Developing into Colorectal Cancer and Endometrial Cancer Based on Gene Expression, Methylation and Mutation Analysis
}

\section{Hongfeng Li}

Tianjin Academy of Traditional Chinese Medicine Affiliated Hospital

\section{Liwei Sun}

Tianjin Huanhu Hospital

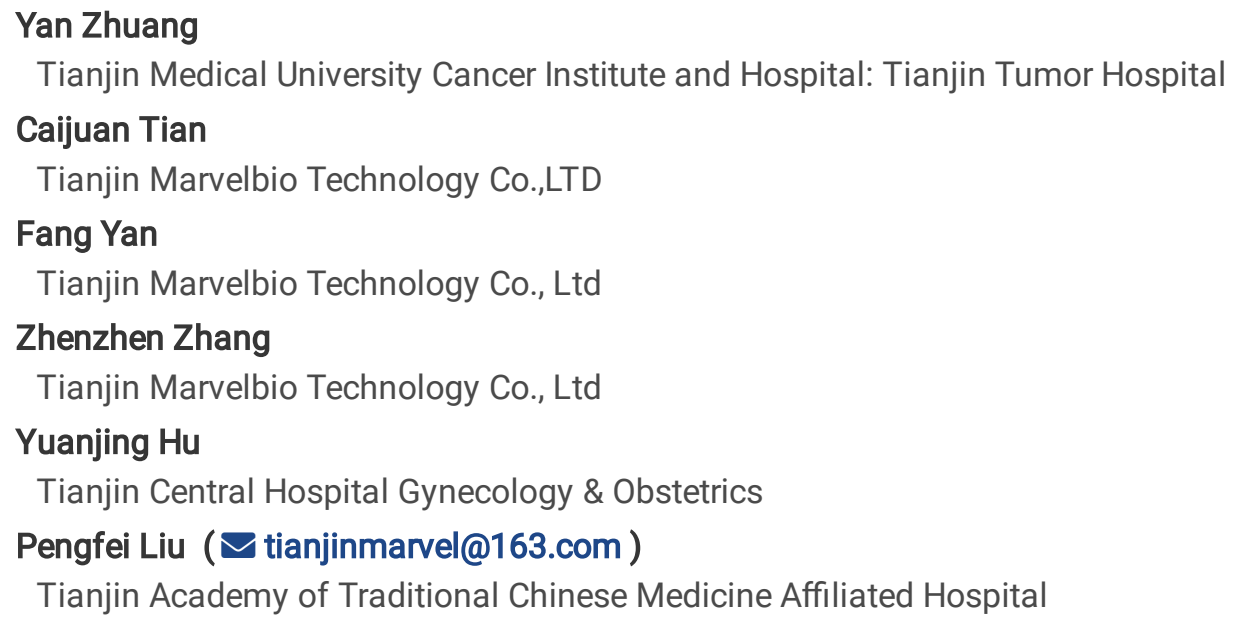

\section{Research Article}

Keywords: Lynch syndrome (LS), colorectal cancer (CRC), endometrial cancer (EC), development mechanism

Posted Date: February 8th, 2022

DOI: https://doi.org/10.21203/rs.3.rs-320385/v1

License: (-) (i) This work is licensed under a Creative Commons Attribution 4.0 International License. Read Full License

Version of Record: A version of this preprint was published at Cancer Causes \& Control on February 11th, 2022. See the published version at https://doi.org/10.1007/s10552-021-01543-w. 


\section{Abstract \\ Purpose}

The aim of this study was to screen biomarkers specific to Lynch syndrome (LS) with colorectal cancer (CRC) or endometrial cancer (EC) to explore the mechanisms by which LS develops into CRC and EC and their differences.

\section{Methods}

Differentially expressed or differentially methylated genes and differential mutations were identified in 10 LS, 50 CRC and 50 EC patients from TCGA, and genes overlapping between LS and CRC or EC (named SGs-LCs and SGs-LEs, respectively) were identified. Afterwards, we annotated the enriched GO terms and pathways and constructed a protein-protein interaction (PPI) network. Finally, samples from 10 clinical cases with MSI-H/MSS CRC and EC were collected to verify the mutations and their correlations with 5 LS pathogenic genes in the SGs-LCs and SGs-LEs.

\section{Results}

A total of 494 SGs-LCs and 104 SGs-LEs were identified and enriched in 106 and 14 GO terms, respectively. There were great differences in the gene count and enriched terms between SGs-LCs and SGs-LEs. In the PPI network, SST, GCG, SNAP25, and NPY had the highest degree of connection among the SGs-LCs, and KIF20A and NUF2 had the highest degree of connection among the SGs-LE. In the SGs-LCs and SGs-LEs, the genes whose expression levels affected the survival of LS, CRC or EC patients were quite different.

\section{Conclusions}

COL11A1 was found to be mutated in MSS CRC patients, similar to the mutations of MSH6. SST, GCG, SNAP25, and NPY may be biomarkers for the development of LS into CRC, and KIF2OA and NUF2 may be markers of LS developing into EC.

\section{Introduction}

Lynch syndrome (LS), also known as hereditary nonpolyposis colorectal cancer (HNPCC), is an autosomal dominant genetic disease caused by defects in DNA mismatch repair (MMR) genes. LS is the most common hereditary colorectal cancer syndrome, with clinical features of early onset and tumour susceptibility in the proximal colon[1, 2]. According to different clinical manifestations, LS can be divided into two categories: LS I and LS II. The only malignant tumour produced by LS I is colorectal cancer (CRC). In LS II, however, in addition to CRC, HNPCC-related parenteral tumours can also occur, such as adenocarcinoma in the stomach, endometrium, pancreas, or bile duct and malignant tumours of the blood system, among others[3]. Approximately 5$6 \%$ of cases of CRC are associated with germline mutations[4]. Patients with hereditary CRC syndromes, such as LS and familial adenomatous polyposis, have a significantly elevated risk of CRC compared with the general population.

LS is the most common cause of hereditary CRC and the only known cause of hereditary endometrial cancer (EC). Approximately $2 \%$ of EC cases are related to LS. EC is the first manifestation of disease in more than $50 \%$ of women with LS. These patients' 10 year risk of developing EC is $26 \%[5]$, and the lifetime risk is $15-60 \%$ [6], which is greater than that of CRC[7]. With the increased popularity and level of detail of genetic testing, the pathogenic genes of LS have been clarified. The main reported genes are MLH1, MSH2, MSH6, PMS2 (MMR genes) and EPCAM (non-MMR gene)[8]. Germline mutations in MMR genes cause microsatellite instability (MSI) and result in the loss of the corresponding MMR protein, thus affecting DNA mismatch repair function and increasing the risk of malignant transformation.

Although the pathogenesis of LS has been gradually elucidated, the molecular mechanism of LS developing into CRC or EC has been less reported, and I few articles have only mentioned that Lynch syndrome patients will develop CRCs as well as some 
endometrial tumors for clinical spectrum overlap[9]. Therefore, this study sought to identify genes specific to LS with CRC or EC by differential expression analysis, differential methylation analysis, gene mutation detection and correlation analysis with the pathogenic genes of LS. Subsequently, through functional enrichment analysis, survival analysis, and clinical sequencing data verification, we clarified the underlying mechanisms of these genes in the development of LS into CRC or EC and their differences. Our study may provide new insights for the early screening and prevention of CRC and EC related to LS.

\section{Materials And Methods}

\section{Data collection and processing}

The transcriptome sequencing data, methylation profiles, tissue mutation data and survival data of 10 LS, 50 CRC and 50 endometrial carcinoma patients were downloaded from The Cancer Genome Atlas (TCGA, https://tcgadata.nci.nih.gov/docs/publications/tcga/) as the training group. The sequencing platform used for the methylation profile was an Illumina HumanMethylation 450 BeadChip.

The transcriptome sequencing data were normalized using the normalize.quantiles function in the preprocessCore V1.32.0 package (http://www.bioconductor.org/packages/3.2/bioc/html/preprocessCore.html), then, log2 transformation was performed, and negative values were taken as 0 . The org.Hs.eg.db package was used to map the Ensembl ID to the gene symbol, and probes without corresponding genes were removed. For duplicate gene IDs, the average expression value was taken as the gene expression value.

\section{Differential expression, differential methylation and mutation analysis}

The differentially expressed genes (DEGs) in LS, CRC and EC were identified with limma V3.32.2

(http://www.bioconductor.org/packages/3.5/bioc/html/limma.html) and recorded as DEGs-L, DEGs-C and DEGs-E, respectively. The threshold criteria were $\mid \log$ (fold-change) $\mid>1$ and $\mathrm{P}<0.05$.

The methylation profiles of LS, CRC and EC were normalized using the preprocessCore V1.32.0 package. Subsequently, a t test was performed on the $\beta$ value of each probe to find probes with $P<0.01$ and $|\Delta \beta|>0.05$. The probes were mapped to the gene symbol using the IlluminaHumanMethylation450kanno.ilmn12.hg19 package

(http://www.bioconductor.org/packages/release/data/annotation/html/llluminaHumanMethylation450kanno.ilmn12.hg19.html), and duplicate gene names were removed. Finally, the differentially methylated genes (DMEs) were obtained and recorded as DMGs-L, DMGs-C and DMGs-E.

The percentage of each mutation type in each group of patients was counted, and the genes with mutation frequencies greater than the median were further screened as high-frequency mutation genes. Genes with two or more mutations in one patient were counted once.

\section{Screening of genes highly related to LS pathogenic genes in DEGs and DMGs}

The DEGs and DMGs highly related to the pathogenic genes of LS (MLH1, MSH2, MSH6, PMS2 and EPCAM) were screened. For the highly related DEGs of LS and CRC, the correlation coefficient between each pathogenic gene and each DEG-L/DEG-C was calculated. Subsequently, the genes with $|r|>$ median(|r|), where $r$ represents the correlation coefficient, were taken as the highly correlated genes. The highly correlated genes in DEGs-L and DEGs-C were intersected to obtain the genes highly correlated with the pathogenic gene in both Lynch syndrome patients and CRC patients. Then, we obtained 5 sets of DEGs with high correlation with the 5 pathogenic genes. These DEGs were combined as DEGs-LC, which were highly related to the 5 pathogenic genes and specific for LS and CRC. Similarly, we acquired DEGs-LE (DEGs from DEGs-L and DEGs-E and specific for LS and EC), DMGs-LC and DMGs-LE. Finally, the DEGs, DMGs and mutations specific to LS and CRC (or EC) were obtained and named SGs-LC (or SGsLE).

\section{Functional enrichment analysis}


Functional and pathway enrichment analyses of SGs-LC and SGs-LE were performed via the Database for Annotation, Visualization and Integrated Discovery (DAVID) V6.7 (http://david.abcc.ncifcrf.gov/) and Kyoto Encyclopedia of Genes and Genomes (KEGG) pathway (http://www.genome.jp/kegg). Gene Ontology (GO) terms and pathways with $\mathrm{P}<0.05$ were selected.

\section{Construction of protein-protein interactive network}

The interacting protein-protein pairs within SGs-LC and SGs-LE were identified via STRING V10.5 (https://string-db.org/). Ultimately, the regulated network was established based on the protein-protein pairs and visualized by Cytoscape V3.5.1 software (http://www.cytoscape.org/download.php). The degree and betweenness of each node were counted by using CentiScaPe (http://apps.cytoscape.org/apps/centiscape), and the node with the largest degree of connectedness was found as the hub node.

\section{Survival analysis}

The survival package in R 3.4.4 was used to calculate the significance of the effect on survival. For the genes with high node degrees in the network, genes were considered highly expressed in the patient when the expression value was greater than the median expression value, otherwise, it was low. Accordingly, the patients were divided into group-high and group-low. The survdiff command[10] was then used to analyse significant differences in the overall survival rate between the two groups. A P value < 0.05 was considered statistically significant.

\section{Validation of the specific DEGs}

MSI was detected in CRC and EC patients. A total of 10 cases of CRC and 10 cases of EC with high-level microsatellite instability (MSI-H) were selected from Tianjin Central Hospital of Gynecology and Obstetrics, Tianjin Huanhu Hospital and Tianjin Medical University Cancer Institute and Hospital, 10 cases of CRC and 10 cases of EC with microsatellite stability (MSS) were also selected. These patients served as the validation group and were then subjected to next-generation sequencing to detect gene mutations. Subsequently, mutations in SGs-LCs and SGs-LEs with high node degrees were verified in these four groups of patients, and their correlation with dislocation mutations in $M L H 1, M S H 2, M S H 6, P M S 2$ and EPCAM was also verified.

Afterwards, Gene Set Enrichment Analysis (GSEA) (https://www.gsea-msigdb.org/gsea/index.jsp) was used to verify the scores of SGs-LC and SGs-LE with high node degree in gene sets related to LS and CRC (LS and EC). Nom P< 0.05 and FDR q $<25 \%$ were the criteria considered to indicate significant differences.

\section{Statistical Analysis}

SPSS Statistics version 21 (SPSS Inc., Chicago, IL, USA) was used for data analysis. Continuous variables were compared between groups by t-test, while categorical variables were expressed as number and frequency and compared by chi-square test or Fisher's exact test. .

\section{Results}

\section{DEGs, DMGs and gene mutations}

A total of 1170 (433 upregulated and 737 downregulated), 1522 (603 upregulated and 919 downregulated) and 1437 (675 upregulated and 762 downregulated) DEGs were obtained in LS, CRC and endometrial carcinoma patients, respectively, and called DEGs-L, DEGs-C and DEGs-E. Figure 1A-C represents the gene expression in total and the heatmap of the top 50 DEGs according to the $\mathrm{P}$ value through cluster analysis. Cluster analysis involves grouping a set of characteristics in such a way that objects in the same group (a cluster) are more similar (in some way) to each other than to the objects in other groups. We found that there was a substantial difference in the 3 sets of DEGs between tumour tissue and normal tissue. Among the above 3 sets of DEGs, there were 653 specific genes for LS and CRC, 35 for LS and EC, and 252 for LS, CRC and EC (Figure 2A).

By analysing the methylation data, we acquired differentially methylated genes (DMGs). A total of 13085 DMGs in LS (DMGsL), 18315 in CRC (DMGs-C) and 18801 in EC (DMGs-E) were obtained. There were 376 DMGs in both LS and CRC, 454 common DMGs in LS and EC, and 12136 overlapping DMGs among the three diseases (Figure 2B). As shown in Figure 2A-B, the number of 
specific DEGs in LS-CRC was significantly higher than that in LS-EC, while the number of specific DMGs in LS-EC was slightly higher than that in LS-CRC, indicating that the pathogenic processes connecting LS to CRC and EC might be different.

In the analysis of the mutation data, we counted the number and types of mutations in three patients (Figure 2C, D). Among the three diseases, missense mutations were the most common, followed by frameshift-del and nonsense mutations (Figure 2C). The differences in mutation type among the 3 diseases included that CRC had a higher proportion of frameshift-del mutations than EC and that the percentage of nonsense mutations was higher in EC than in CRC, illustrating that CRC and EC may occur and develop through different types of mutations.

We further counted the common mutations whose mutation frequency was greater than the median (median mutation of LS and CRC was 1 and EC mutation was 2). There were 111 high-frequency mutations in LS, 4563 in CRC and 2883 in EC. The common mutated genes in LS and CRC were screened, and the genes mutated in EC were excluded. Thus, specific highfrequency mutated genes in LS and CRC (Mut-LC) were obtained, with a total of 19 genes. Similarly, 11 common mutated genes were obtained in LS and EC (Mut-LE) (Figure 2D). The number of specific mutations in both groups was small.

\section{Identification of DEGs and DMGs highly related to LS pathogenic genes}

A total of 460 DEGs in both DEGs-L and DEGs-C (DEGs-LC) were obtained by analysing the correlation between DEGs and the 5 LS pathogenic genes, as well as 24 DEGs in DEGs-L and DEGs-E (DEGs-LE). In the three groups of DMGs, we obtained 15 specific DMGs (DMGs-LC) of LS and CRC and 64 specific DMGs (DMGs-LE) of LS and EC, which were highly correlated with 5 LS pathogenic genes.

Then, DEGs-LC, DMGs-LC and Mut-LC (DEGs-LE, DMGs-LE and Mut-LE) were combined, and duplicate genes were deleted. Ultimately, 494 specific genes for LS and CRC (SGs-LC) were obtained, including PTCHD1, SYT4, and COPDA1, and 99 LS and endometrial specific genes (SGs-LE) were obtained, including CDC20B, SLC10A4, and LY6K (Supplementary Table 1). The top 20 SGs-LC and SGs-LE according to $P$ value are listed in Table 1. The numbers of SGs-LC and SGs-LE were quite different. Thus, it is speculated that LS might develop into CRC in more complex ways.

\section{The enriched GO terms and pathways}

After enrichment analysis, SGs-LCs were found to be enriched in 7 KEGG pathways and $106 \mathrm{GO}$ terms, including 60 biological processes (BPs), 22 cellular components (CCs), and 24 molecular functions (MFs). The main enriched BPs were feeding behaviour, collagen catabolic process, synapse organization and regulation of appetite. Enriched CCs were extracellular space, plasma membrane, anchoring component of membrane, etc. Enriched MFs were hormone activity, neuropeptide hormone activity, serotonin-activated cation-selective channel activity and bile acid transmembrane transporter activity. Figure $3 \mathrm{~A}$ shows the top 20 GO terms according to $P$ value. The KEGG pathways of SGs-LCs were significantly enriched in serotonergic synapse, maturity onset diabetes of the young, PPAR signalling pathway and arrhythmogenic right ventricular cardiomyopathy (ARVC) (Table 2).

SGs-LEs were enriched in $12 \mathrm{GO}$ terms, including $5 \mathrm{BPs}, 6 \mathrm{CCs}$ and $1 \mathrm{MF}$. The $\mathrm{GO}$ terms are shown in Figure 3B, among which peroxisome,,mitochondrion, protein transport, cellular response to DNA damage stimulus and membrane had $\mathrm{P}$ values less than 0.05. The only KEGG pathway identified as enriched among the SGs-LEs was the peroxisome pathway, however, the enrichment was not statistically significant $(P=0.050$, Table 2$)$. There was a great difference in enriched GO terms and pathways between SGs-LCs and SGs-LEs, and SGs-LCs were enriched in more pathways, indicating that LS might likely develop into CRC through more pathways, consistent with our previous speculation.

\section{Protein-protein interaction networks}

After STRING analysis, 663 interaction pairs between 280 proteins were obtained in SGs-LCs. These pairs formed 12 clusters and contained 66 genes (Figure 4A). SNAP25,SST,GCG and GABRG2 were involved in most pairs. Table 3A shows the top 20 genes with the highest degrees in the network. A total of 24 interactions were obtained from SGs-LEs. In the whole network, there were 3 clusters containing 11 genes with degree $\geq 2$ (Figure 4B), of which KIF20A and NUF2 had the highest degrees (Table 3B). These genes could be potential key genes for the development of LS into CRC or EC. 


\section{Correlation between gene expression level and survival}

In SGs-LCs, the genes with a significant difference in survival rate among LS patients with high expression levels and low expression levels were ELAVL3 $(\mathrm{P}=0.013), \operatorname{ALPI}(\mathrm{P}=0.020), \operatorname{GCGR}(\mathrm{P}=0.020), \operatorname{HS6ST3}(\mathrm{P}=0.032), C N G B 1(\mathrm{P}=0.033)$ and $R O R B(\mathrm{P}=0.047)$ (Figure 5A-F). The expression levels of $C A 10(\mathrm{P}=0.008), \operatorname{HTR} 4(\mathrm{P}=0.028), N R A P(\mathrm{P}=0.031), C L D N 19(\mathrm{P}=$ 0.041), $C O L 18 A 1(P=0.047), S M K R 1(P=0.039)$ and $T P H(P=0.0027)$ were significantly correlated with the survival rates of $C R C$ patients (Figure 5G-M). In SGs-LEs, there was no significant difference in survival rates between LS patients with high and low expression of any SGs-LE gene. In EC, the genes with significantly different survival rates between patients with high and low expression levels were $C D C 45(\mathrm{P}=0.015), W D R 31(\mathrm{P}=0.024)$ and $U Q C R Q(P=0.037)$ (Figure $5 \mathrm{~N}-\mathrm{P})$. These genes might be key genes for the prognosis of patients with LS, CRC and EC.

\section{Verification of the specific genes}

MSI is caused by a defect in one of the MMR genes and is strongly related to tumorigenesis. The MMR genes $(M L H 1, M S H 2$, MSH6, and PMS2) are pathogenic genes in LS. Therefore, we first analysed the mutations in SGs-LCs and SGs-LEs in CRC and EC patients with MSI-H and MSS. COL11A1 is associated with malignancy in colorectal cancer. In this study, COL11A1 was identified in SGs-LC and exhibited one missense mutation, three frameshift mutations and one intron mutation in MSS CRC patients. In addition, the mutation profile of $C O L 11 A 1$ was very similar to that of $M S H 6$, as both contained intron, missense and frameshift mutations, therefore, COL11A1 may be the key gene to distinguish MSI-H and MSS CRC patients.

Finally, we analysed the expression of SGs-LC and SGs-LG in normal and tumour samples in LS-, CRC-, and EC-related pathways through GSEA of GO gene sets. SGs-LE was not significantly different between normal and tumour samples. The differentially expressed genes in SGs-LC were enriched in 4 GO terms:HP_clinical_course,GOMF_ion_transmembrane_transporter_activity, GOBP_neurogenesis,GOBP_neuron_differentiatiation(Figure 6 A-D).

\section{Discussion}

LS is an autosomal dominant disease. Carriers of pathogenic MMR mutations have a lifetime risk of approximately $30-70 \%$ for $\mathrm{CRC}$, and the risk of EC in female carriers is approximately 30-60\%[11]. The risk of other malignant tumours, such as gastric cancer, pancreatic cancer and urinary system tumours, is also significantly higher in MMR mutation carriers than that in the general population[12,13]. Patients with LS are at higher risk of gastrointestinal, gynaecological, skull and skin cancers, the most common of which are CRC and EC.

Although some progress has been made in the mutation of MMR genes in CRC and EC, the molecular mechanism and comparison of LS to CRC or EC has not been reported[14]. In this study, we analysed the differential expression, differential methylation and mutation of genes in LS, CRC and EC from the TCGA database. There were significantly more specific DEGs in LS and CRC than in LS and EC. However, the specific DMGs showed the opposite result, indicating that the development of LS into CRC may occur through differential gene expression, while the development into EC may occur through gene methylation (Figure 2A, B). Specific mutations were less frequent in both LS-CRC and LS-EC, and the proportion of mutation types was significantly different in LS, CRC and EC patients; it was indicated that missense mutation may have some influence on the development of LS into cancers but was not the main mechanism (Figure 2C, D).

By analysing the correlation of DEGs, DMGs and mutations with the LS pathogenic genes $M L H 1, M S H 2, M S H 6, P M S 2$ and EPCAM, we finally obtained 493 specific genes for LS and CRC (SGs-LC) and 99 specific genes for LS and EC (SGs-LE). The results of GO enrichment analysis showed that SGs-LCs and SGs-LEs were enriched in different GO terms and KEGG pathways, and the numbers were quite different. Although the enriched pathways of SGs-LCs and SGs-LEs were different, they both included pathways related to peroxisomes, which revealed that there might be correlations between the mechanisms of LS developing into $\mathrm{CRC}$ and EC as well as certain differences. These results provide insights into the molecular mechanism by which LS progresses to $\mathrm{CRC}$ or EC.

Through the construction of the PPI network, we obtained 4 (SNAP25, SST, GSG, GSBRG2) and 2 (NUF2,KIF20A) genes with high node degrees in SGs-LC and SGs-LE, respectively. SST (somatostatin) was isolated and purified from the sheep hypothalamus in 
1973. Subsequent studies have confirmed that somatostatin not only exists in the hypothalamus but is also widely distributed in the brain, pancreas and intestinal nerve cells. SST is a regulatory peptide that functions in exocrine, endocrine, paracrine and autocrine processes. It has a wide range of biological activities and plays an important role in regulating the physiological functions and pathogenesis of some diseases. SST can not only inhibit the hormone production of endocrine cells but also inhibit the mitosis of cells. Most experimental animals and human cell lines have shown that SST can inhibit the growth of tumours such as pancreatic cancer, CRC and liver cancer[15, 16]. NPY (neuropeptide $Y$ ) is a sympathetic neurotransmitter belonging to the pancreatic polypeptide family, and a single nucleotide polymorphism (SNP) associated with this gene is related to hypertension, obesity, diabetes and cardiovascular disease[17]. KIF20A (Kinesin 20A), a member of the kinesin superfamily, is mainly involved in mitosis[18]. Recently, abnormal KIF20A expression has been found in breast cancer, cervical squamous cell carcinoma and hepatocellular carcinoma, suggesting that this gene is closely related to the formation and development of tumours[19-22]. NUF2, a cell division-related gene, plays a role in stabilizing centromeres and ensuring accurate separation of chromosomes during mitosis[23]. Studies have found that NUF2 is highly expressed in a variety of malignant tumours, including lung cancer, liver cancer, CRC, and gastric cancer, and plays an important role in tumour formation and development[24-26]. These genes may be the key genes for the development of LS into CRC and EC.

Furthermore, we analysed the effect of SGs-LCs and SGs-LEs on patient survival. After screening, we found that the expression levels of different genes in LS, CRC and EC patients were quite different, and the genes affecting the survival of patients were also different. The expression levels of ALPI, CNGB1, ELAVL3, GCGR, HS6ST3, and RORB in SGs-LC were significantly correlated with the survival rate of patients with LS and CA10,CLDN19, COL18A1, HTR4,SMKR1,TPH1 and NRAP with CRC. In SGs-LE, there was a significant difference in the survival rate between endometrial carcinoma patients with high and low expression levels of CDC45, UQCRQ and WDR31. Previous studies have reported some of these genes in cancer. Takashi et al.[27] detected GCGR expression in CRC tissues, and downstream signals of GCGR, including AMPK and MAPK pathways, play vital roles in the proliferation of CRC in vitro and in vivo. HTR4 performs an important function in human prostate cancer through 5-HT secreted by cancerous tumours and mast cells surrounding the tumours. In addition, in prostatic cancer, oestrogen may regulate the effect of HTR4 through oestrogen receptor $\beta$ (ER $\beta$ ) to affect the development of cancer[28]. COL 18A1 encodes XVIII collagen, which is a widely expressed nonfibrillar collagen and an important component of the extracellular matrix (ECM), which was consistent with our enrichment analysis finding that $C O L 18 A 1$ was enriched in the $\mathrm{GO}$ term of extracellular space. A study found that the risk of sporadic breast cancer was significantly increased in patients with the COL18A1 D104N polymorphism[29]. In addition, the upregulated expression of COL18A1 was found in bladder cancer patients with tumour stages T1 and T2, which may be involved in the progression of bladder cancer by affecting extracellular matrix-receptor interactions and adhesion sites[30]. CDC45 is a component of the cell division cycle 45 (CDC45) minichromosome maintenance protein complex (MCM) and has been reported to be upregulated and identified as a hub gene in non-small-cell lung cancer[31]. CDC45 might also promote papillary thyroid cancer progression and nasopharyngeal carcinoma[32, 33]. These genes may be potential prognostic markers for LS, CRC and EC.

Finally, we compared the mutations of these genes in CRC and EC patients with MSI-H and MSS and their correlation with dislocation mutations in five pathogenic genes of LS. Microsatellites are short tandem repeats that are present throughout the human genome. The length of microsatellites changes in tumour cells with respect to that in normal cells due to the insertion or deletion of repeat units; this process is called MSI. Numerous studies have shown that MSI is caused by defects in MMR genes and is closely related to tumorigenesis. MSI has been clinically used as an important molecular marker for the prognosis and adjuvant treatment of CRC and other solid tumours and has been used to assist in screening for LS. In this study, COL11A1 exhibited 1 missense, 3 frameshift, and 1 intron mutation in MSS CRC. The correlation analysis results showed that COL 11A1 mutation spectrum was consistent with that of $\mathrm{MSH} 6$ mutations and had a strong correlation with MSH6 mutation. The COL 11A1 gene is located in the chromosome 1p21 region, contains 68 exons, and is mainly expressed in articular cartilage. Recent studies have found that COL 11A1 plays an important role in the occurrence and development of tumours, and its expression is upregulated in head and neck squamous cell carcinoma and in ovarian, gastric, and pancreatic cancer[34-37]. Our results illustrated that COL11A1 may be a marker for the differentiation of MSI-H and MSS CRC patients and for the development of LS into MSS CRC. In addition, all of the four validation GO terms enriched in SGs-LCs in GSEA database are related to CRC... Our results revealed few verified genes and GO terms. The possible reasons for this are as follows: first, the number of patients we collected was small, and the gene mutation detection was not comprehensive; second, most of the data in TCGA are from Caucasian populations, whereas the patients we analysed were all from Asian populations. The difference in race may also be 
one of the reasons for the difference in mutation type and frequency. More experimental research is needed to confirm the current findings.

In brief, we obtained genes specific to LS-CRC and LS-EC, which may be key genes involved in the progression of LS to CRC and EC. At the same time, our study reveals that the molecular mechanisms of LS development to CRC and EC are different. Since cancer is the result of multiple highly complex molecular mechanisms, a single pathway is not sufficient to explain cancer pathogenesis[38]. However, our findings provide novel insights into the mechanisms by which LS develops into CRC or EC and their differences.

\section{Declarations}

\section{Funding}

The work was supported by Tianjin Science and Technology Plan Project (17ZXMFSY00160).

\section{Conflicts of Interest}

The authors have no conflicts of interest to declare.

\section{Availability of data and materials}

The datasets used and/or analyzed during the current study are available from the corresponding author on reasonable request.

\section{Author Contributions}

Hongfeng Li and Liwei Sun designed experiments and wrote the manuscript. Yan Zhuang collected data. Caijuan Tian, Fang Yan and Zhenzhen Zhang analysed and interpreted the data. Yuanjing Hu and Pengfei Liu reviewed the manuscript. Yuanjing Hu and Pengfei Liu given the version to be published and agreed to be accountable for all aspects of the work. All authors read and approved the final manuscript.

\section{Ethics approval and consent to participate}

Informed consent had been obtained from the participants. This research was approved by the Ethical Committee of Tianjin Central Hospital of Gynecology \& Obstetrics, Tianjin Huanhu Hospital and Tianjin Medical University Cancer Institute and Hospital.

\section{References}

1. Schneider NB, Pastor T, Paula AE, Achatz MI, Santos ÂRD, Vianna FSL et al (2018) Brazilian Lynch Syndrome Study Group. Germline MLH1, MSH2 and MSH6 variants in Brazilian patients with colorectal cancer and clinical features suggestive of Lynch Syndrome. Cancer Med 7(5):2078-2088

2. Tiwari AK, Roy HK, Lynch HT (2016) Lynch syndrome in the 21st century: clinical perspectives. QJM 109(3):151-158

3. Biller LH, Syngal S, Yurgelun MB (2019) Recent advances in Lynch syndrome. Fam Cancer 18(2):211-219

4. Oh BY (2017) Hereditary Nonpolyposis Colorectal Cancer. Semin Surg Oncol 18:305-313

5. Ryan NAJ, Blake D, Cabrera-Dandy M, Glaire MA, Evans DG, Crosbie EJ (2018) The prevalence of Lynch syndrome in women with endometrial cancer: A systematic review protocol. Syst Rev 7(1):121

6. Pathak SJ, Mueller JL, Okamoto K, Das B, Hertecant J, Greenhalgh L et al (2019) EPCAM mutation update: Variants associated with congenital tufting enteropathy and Lynch syndrome. Hum Mutat 40(2):142-161

7. Obermair A, Youlden DR, Young JP, Young JP, Lindor NM, Baron JA et al (2010) Risk of endometrial cancer for women diagnosed with HNPCC -related colorectal carcinoma. Int J Cancer 127(11):2678-2684

8. D'Arcy BM, Blount J, Prakash A (2019) Biochemical and Structural Characterization of Two Variants of Uncertain Significance in the PMS2 Gene. Hum Mutat 40(4):458-471 
9. Carethers JM (2015) Biomarker-directed Targeted Therapy in Colorectal Cancer. J Dig Cancer Rep 3(1):5-10

10. Harrington DP, Fleming TR (1982) A class of rank test procedures for censored survival data. Biometrika 69:553-566

11. Shia J, Klimstra DS, Nafa K, Offit K, Guillem JG, Markowitz AJ et al (2005) Value of immunohistochemical detection of DNA mismatch repair proteins in predicting germline mutation in hereditary colorectal neoplasms. Am J Surg Pathol 29(1):96104

12. Giuffre G, Muller A, Brodegger T, Bocker-Edmonston T, Gebert J, Kloor M et al (2005) Microsatellite analysis of hereditary nonpolyposis colorectal cancer-associated colorectal adenomas by laser-assisted microdissection: correlation with mismatch repair protein expression provides new insights in early steps of tumorigenesis. J Mol Diagn 7(2):160-170

13. Muller A, Beckmann C, Westphal G, Edmonston TB, Friedrichs N, Dietmaier W et al (2006) Prevalence of the mismatch-repairdeficient phenotype in colonic adenomas arising in HNPCC patients: results of a 5-year follow-up study. Int J Colorectal Dis 21(7):632-641

14. Chen W, Frankel WL (2019) A practical guide to biomarkers for the evaluation of colorectal cancer. Mod Pathol 32:1-15

15. Schmid H, Jensen M (2009) Abstract \#808: Inhibitory effect of pasireotide (SOM230) in a rat pancreatic tumor model (CA20948) alone and in combination with everolimus (RAD001). Cancer Res 69:808

16. Liu Y, Chew MH, Tham CK, Tang CL, Ong SY, Zhao Y (2016) Methylation of serum SST gene is an independent prognostic marker in colorectal cancer. Am J Cancer Res 6(9):2098-2108

17. Shah SH, Freedman NJ, Zhang LS, Crosslin DR, Stone DH, Haynes C et al (2009) Neuropeptide Y gene polymorphisms confer risk of early-onset atherosclerosis. PloS Genet 5(1):e1000318

18. Sarli VC, Giannis A (2006) Inhibitors of Mitotic Kinesins: Next Generation Antimitotics. ChemMedChem 1(3):293-298

19. Stangel D, Erkan M, Buchholz M, Gress T, Michalski C, Raulefs S et al (2015) KIF20A inhibition reduces migration and invasion of pancreatic cancer cells. J Surg Res 197(1):91-100

20. Khongkow P, Gomes AR, Gong C, Man EPS, Tsang JWH, Zhao F et al (2016) Paclitaxel targets FOXMI to regulate KIF20A in mitotic catastrophe and breast cancer paclitaxel resistance. Oncogene 35(8):990-1002

21. Zhang WJ, He WL, Shi YJ, Gu HF, Li M, Liu ZM et al (2016) High expression of KIF20A is associated with poor overall survival and tumor progression in early-stage cervical squamous cell carcinoma. PLoS ONE 11(12):el67449

22. Shi C, Huang DL, Lu NH, Chen D, Zhang MH, Yan YH et al (2016) Aberrantly activated Gli2-KIF20A axis is crucial for growth of hepatocellular carcinoma and predicts poor prognosis. Oncotarget 7(18):26206-26219

23. Liu D, Ding X, Du J, Cai X, Huang YJ, Ward T et al (2007) Human NUF2 Interacts with Centromere-associated Protein E and Is Essential for a Stable Spindle Microtubule-Kinetochore Attachment. J Biol Chem 282(2):21415-21424

24. Liu Q, Dai SJ, Li H, Dong L, Peng YP (2014) Silencing of NUF2 inhibits tumor growth and induces apoptosis in human hepatocellular carcinomas. Asian Pac J Cancer Prev 15(20):8623-8629

25. Kaneko N, Miura K, Gu Z, Karasawa H, Ohnuma S, Sasaki H et al (2009) siRNA-mediated knockdown against CDCA1 and KNTC2, both frequently overexpressed in colorectal and gastric cancers, suppresses cell proliferation and induces apoptosis. Biochem Biophys Res Commun 390(4):1235-1240

26. Hu P, Shangguan J, Zhang L (2015) Downregulation of NUF2 inhibits tumor growth and induces apoptosis by regulating IncRNA AF339813. Int J Clin Exp Pathol 8(3):2638-2648

27. Takashi Y, Eiji K, Hiroyuki K, Tanaka T, Kataoka H, Imaeda K et al (2018) Glucagon promotes colon cancer cell growth via regulating AMPK and MAPK pathways. Oncotarget 9(12):10650-10664

28. Nakamura Y, Ise K, Yamazaki Y, Fujishima F, McNamara KM, Sasano H (2017) Serotonin receptor 4 (5-hydroxytryptamine receptor Type 4) regulates expression of estrogen receptor beta and cell migration in hormone-naive prostate cancer. Indian $\mathrm{J}$ Pathol Microbiol 60(1):33-37

29. LourençO GJ, Cardoso-Filho C, GonçAles NSL, Shinzato JY, Zeferino LC, Nascimento H et al (2006) A high risk of occurrence of sporadic breast cancer in individuals with the 104NN polymorphism of the COL18A1 gene. Breast Cancer Res Treat 100(3):335-338

30. Fang ZQ, Zang WD, Chen R, Wang XW, Yi SH, Chen W et al (2013) Gene expression profile and enrichment pathways in different stages of bladder cancer. Genet Mol Res 12(2):1479-1489

Page 9/18 
31. Huang JB, Li Y, Lu ZL, Che Y, Sun SG, Mao SS et al (2019) Analysis of functional hub genes identifies CDC45 as an oncogene in non-small cell lung cancer - a short report. Cell Oncol 42(4):571-578

32. Sun J, Shi R, Zhao S, Li XN, Lu S, Bu HM, Ma XH (2017) Cell division cycle 45 promotes papillary thyroid cancer progression via regulating cell cycle. Tumor Biol 39(5):1010428317705342

33. Li MY, Ma CX, Wu HX, Wu XH, Yu JK (2018) Gene Differential Expression and Protein Interaction Networks Analysis in Nasopharyngeal Cancer. Letters In Biotechnology 29:345-350. (In Chinese)

34. Wu YH, Chang TH, Huang YF, Chen CC, Chou CY (2015) COL11A1 confers chemoresistance on ovarian cancer cells through the activation of Akt/c/EBPß pathway and PDK1 stabilization. Oncotarget 6(27):23748-23763

35. Li AQ, Li J, Lin JP, Zhou W, Si JM (2017) COL11A1 is overexpressed in gastric cancer tissues and regulates proliferation, migration and invasion of HGC-27 gastric cancer cells in vitro. Oncol Rep 37(1):333-340

36. Sok JC, Lee JA, Dasari S, Contrucci SC, Egloff AM, Trevelline BK et al (2013) Collagen type XI a1 facilitates head and neck squamous cell cancer growth and invasion. Br J Cancer 09(12):3049-3056

37. García-Pravia C, Galván JA, Gutiérrez-Corral N, Solar-García L, García-Pérez E, García-Ocaña M et al (2013) Overexpression of COL11A1 by Cancer-Associated Fibroblasts: Clinical Relevance of a Stromal Marker in Pancreatic Cancer. PLoS ONE 8(10):e78327

38. Cao L, Chen Y, Zhang M, Xu DQ, Liu Y, Liu TL et al (2018) Identification of hub genes and potential molecular mechanisms in gastric cancer by integrated bioinformatics analysis. PeerJ 6:e5180

\section{Tables}

Table 1 The top 20 SGs-LC and SGs-LE according to P value 


\begin{tabular}{|c|c|c|c|c|c|c|}
\hline Group & Gene & $\log \mathrm{FC}$ & P Value & Gene & $\log \mathrm{FC}$ & P Value \\
\hline \multirow[t]{10}{*}{ SGs-LC } & AADACL2 & -3.104 & $1.88 \mathrm{E}-16$ & $\mathrm{CDH} 10$ & -2.608 & 1.60E-09 \\
\hline & LINC00507 & -2.998 & 2.32E-16 & PCAT18 & -2.674 & 1.70E-09 \\
\hline & DHRS7C & -3.118 & $6.95 \mathrm{E}-13$ & LINC02616 & -2.736 & $1.38 \mathrm{E}-08$ \\
\hline & LINC00460 & 3.325 & $1.04 \mathrm{E}-11$ & SYT10 & -2.551 & $1.65 \mathrm{E}-08$ \\
\hline & KRT24 & -3.688 & $1.18 \mathrm{E}-11$ & SPATA12 & 1.586 & 1.87E-08 \\
\hline & VSTM2A-OT1 & -2.434 & $2.16 \mathrm{E}-10$ & TM4SF19 & 2.436 & $2.40 \mathrm{E}-08$ \\
\hline & CST2 & 3.382 & 2.61E-10 & MPP4 & 2.438 & $2.76 \mathrm{E}-08$ \\
\hline & HMX3 & -2.567 & 4.64E-10 & CA7 & -1.676 & $3.41 \mathrm{E}-08$ \\
\hline & NPY2R & -3.139 & 8.97E-10 & LINC02023 & -2.826 & 3.67E-08 \\
\hline & KHDRBS2 & -2.859 & 1.39E-09 & ZNF492 & -2.531 & 3.83E-08 \\
\hline \multirow[t]{10}{*}{ SGs-LE } & LINC02691 & -1.925 & 1.01E-05 & BHLHA15 & -1.035 & 0.002 \\
\hline & MIR27B & -1.731 & 1.67E-05 & ZPBP & 1.596 & 0.002 \\
\hline & LY6K & -1.820 & $5.84 \mathrm{E}-05$ & HAPLN1 & -1.226 & 0.003 \\
\hline & IGF2-AS & 2.134 & $6.39 \mathrm{E}-05$ & CLPSL2 & 1.265 & 0.003 \\
\hline & SLC10A4 & -1.106 & $2.98 \mathrm{E}-04$ & LSMEM2 & -1.144 & 0.003 \\
\hline & ADAMTS9-AS2 & -1.282 & 4.56E-04 & ACKR4 & -1.184 & 0.006 \\
\hline & $\mathrm{H} 2 \mathrm{AC} 13$ & -1.175 & $5.24 \mathrm{E}-04$ & LOC101927972 & 1.392 & 0.007 \\
\hline & $\mathrm{ERICH} 4$ & 1.660 & 8.61E-04 & LOC112267895 & 1.044 & 0.008 \\
\hline & CDC20B & 1.241 & $9.52 \mathrm{E}-04$ & ТВX20 & 1.523 & 0.008 \\
\hline & $A B C D 2$ & -1.359 & 0.001 & LOC105373878 & -1.070 & 0.009 \\
\hline
\end{tabular}

SGs-LC, specific genes in Lynch syndrome and colorectal cancer; SGs-LE, specific genes in Lynch syndrome and endometrial cancer

Table 2 SGs-LC and SGs-LE enriched KEGG pathways

\begin{tabular}{|llll|}
\hline Group & Pathway & Count & P Value \\
\hline \multirow{3}{*}{ SGs-LC } & hsa04726:Serotonergic synapse & 9 & 8.32 E-04 \\
\cline { 2 - 4 } & hsa04950:Maturity onset diabetes of the young & 4 & 0.011 \\
\cline { 2 - 4 } & hsa03320:PPAR signaling pathway & 5 & 0.032 \\
\cline { 2 - 4 } & hsa05412:Arrhythmogenic right ventricular cardiomyopathy (ARVC) & 5 & 0.032 \\
\cline { 2 - 4 } & hsa04080:Neuroactive ligand-receptor interaction & 10 & 0.062 \\
\cline { 2 - 4 } & hsa04970:Salivary secretion & 5 & 0.069 \\
\cline { 2 - 4 } & hsa04974:Protein digestion and absorption & 5 & 0.074 \\
\hline SGs-LE & hsa04146:Peroxisome & 3 & 0.050 \\
\hline
\end{tabular}


SGs-LC, specific genes in Lynch syndrome and colorectal cancer; SGs-LE, specific genes in Lynch syndrome and endometrial cancer

Table 3 The nodes of the PPI network with high degree in Lynch syndrome-colorectal cancer and Lynch syndrome-endomemetrial cancer specific genes

Gene Degree Gene Degree

A. Top 20 nodes in Lynch syndrome-colorectal cancer specific genes

\begin{tabular}{llll}
\hline SNAP25 & 33 & GAD1 & 17 \\
\hline SST & 27 & SOX2 & 17 \\
\hline GCG & 26 & ASCL1 & 16 \\
\hline GABRG2 & 26 & ELAVL3 & 16 \\
\hline NEUROD1 & 21 & NEUROG3 & 15 \\
\hline CHGA & 21 & ELAVL4 & 15 \\
\hline SYT4 & 21 & PYY & 14 \\
\hline CA10 & 20 & OLFM3 & 13 \\
\hline NPY & 18 & TPH1 & 13 \\
\hline SNCB & 18 & CHAT & 12
\end{tabular}

B. 8 nodes with degree $\geq 2$ in Lynch syndrome-endomemetrial cancer specific genes

\begin{tabular}{llll}
\hline NUF2 & 3 & RPL17 & 3 \\
\hline KIF20A & 3 & CDC45 & 2 \\
\hline COA6 & 3 & NDUFC1 & 2 \\
\hline MRPS12 & 3 & UQCRQ & 2 \\
\hline RPL15 & 3 & LY6K & 2 \\
\hline WDR31 & 3 & &
\end{tabular}

PPI, protein-protein interaction

\section{Figures}



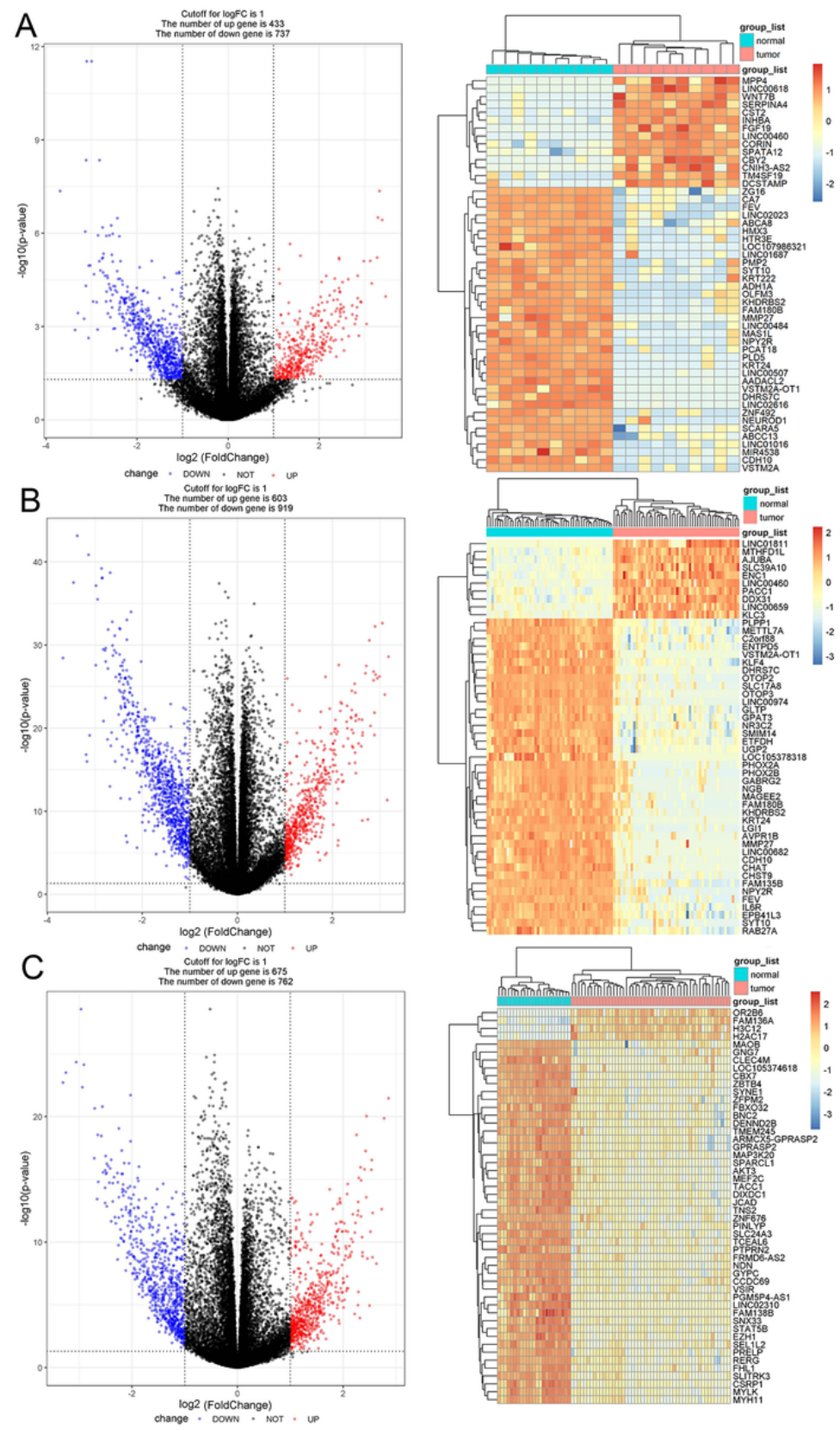

Figure 1

Cluster analysis of DEGs in Lynch syndrome, colorectal cancer and endometrial cancer.

The volcano plot and heatmap of DEGs-L (A), DEGs-C (B) and DEGs-E (C) showed that the expression of the 3 sets of DEGs was significantly different between tumour and normal tissues. Blue, red and black spots represent downregulated, upregulated and nonsignificantly expressed genes, respectively.

DEGs, differentially expressed genes, DEGs-L, DEGs in Lynch syndrome, DEGs-c, DEGs in colorectal cancer, DEGs-E, DEGs in endometrial cancer 
A

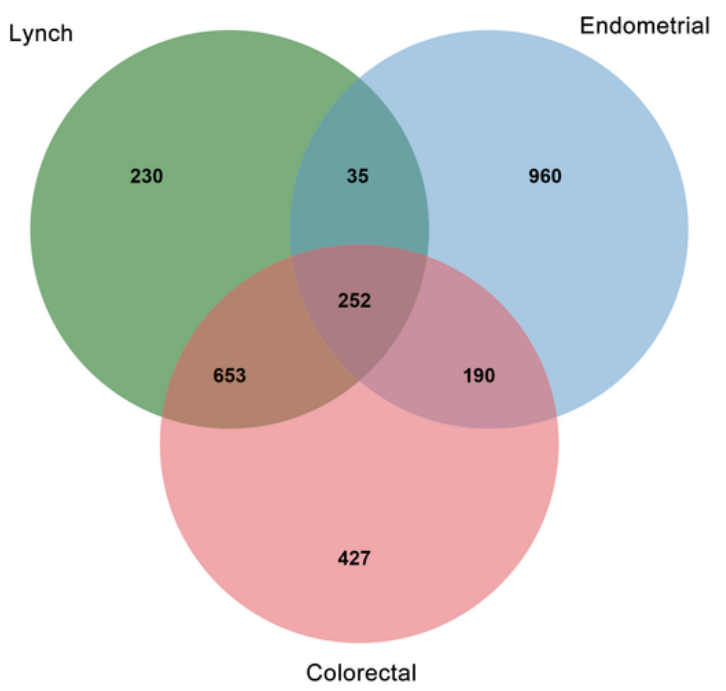

\section{C}

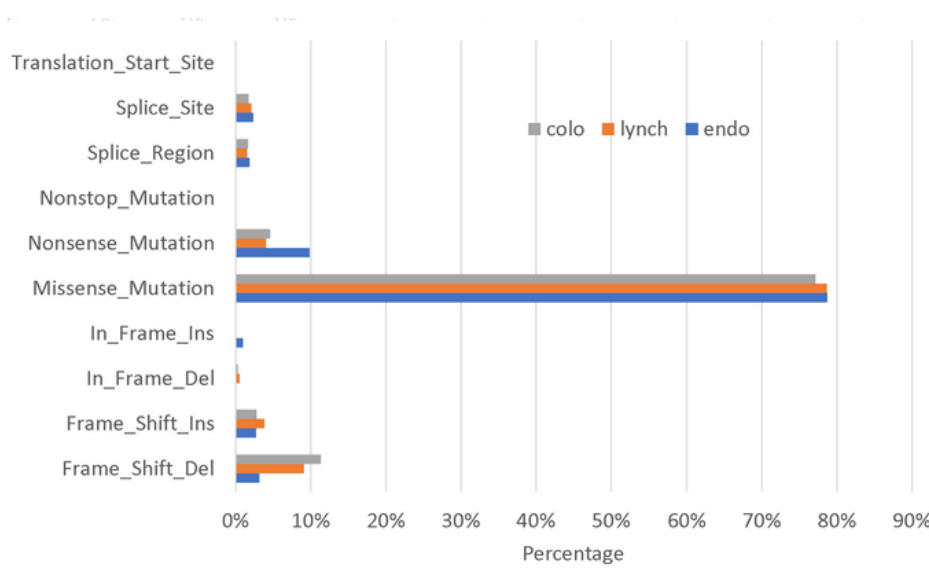

B

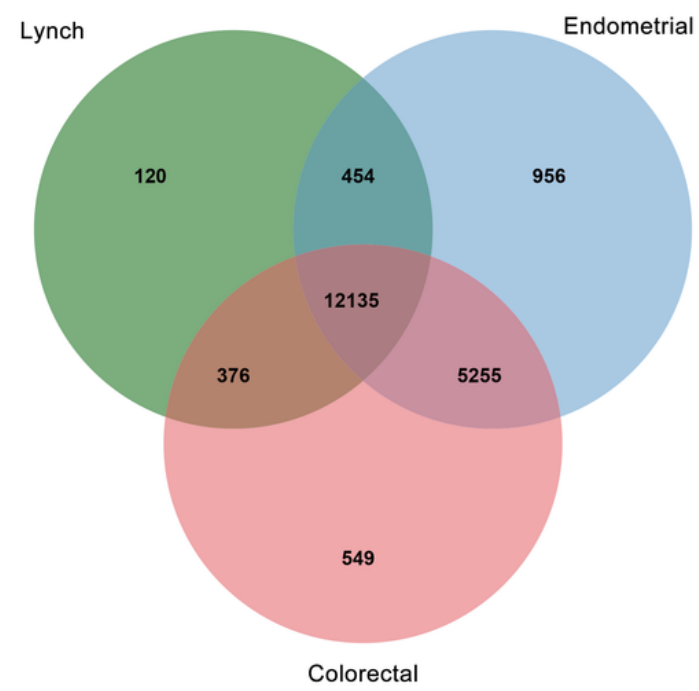

D

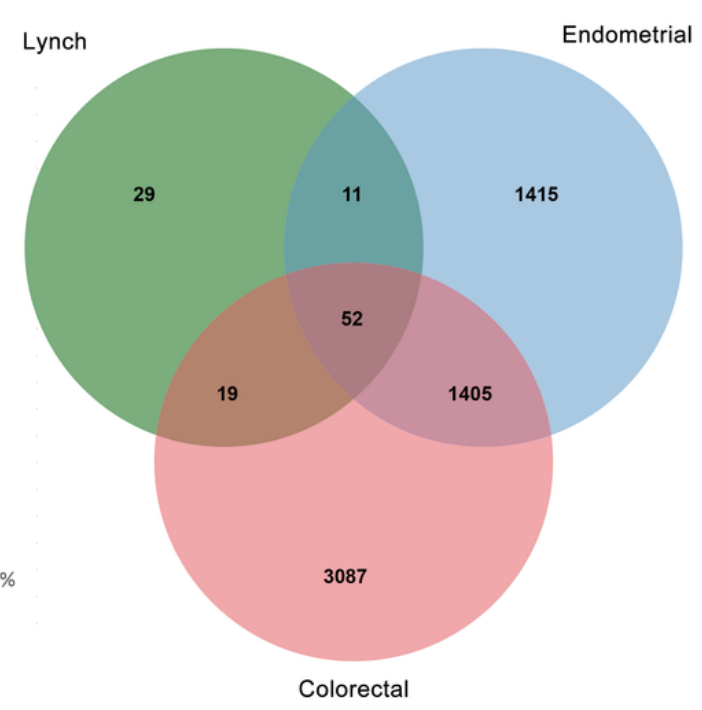

\section{Figure 2}

Venn diagrams of DEGs (A), DMGs (B), mutations (D), and the percentages of mutation types (C) in Lynch syndrome, colorectal cancer, and endometrial cancer

DEGs, differentially expressed genes, DMGs, differentially methylated genes 
A

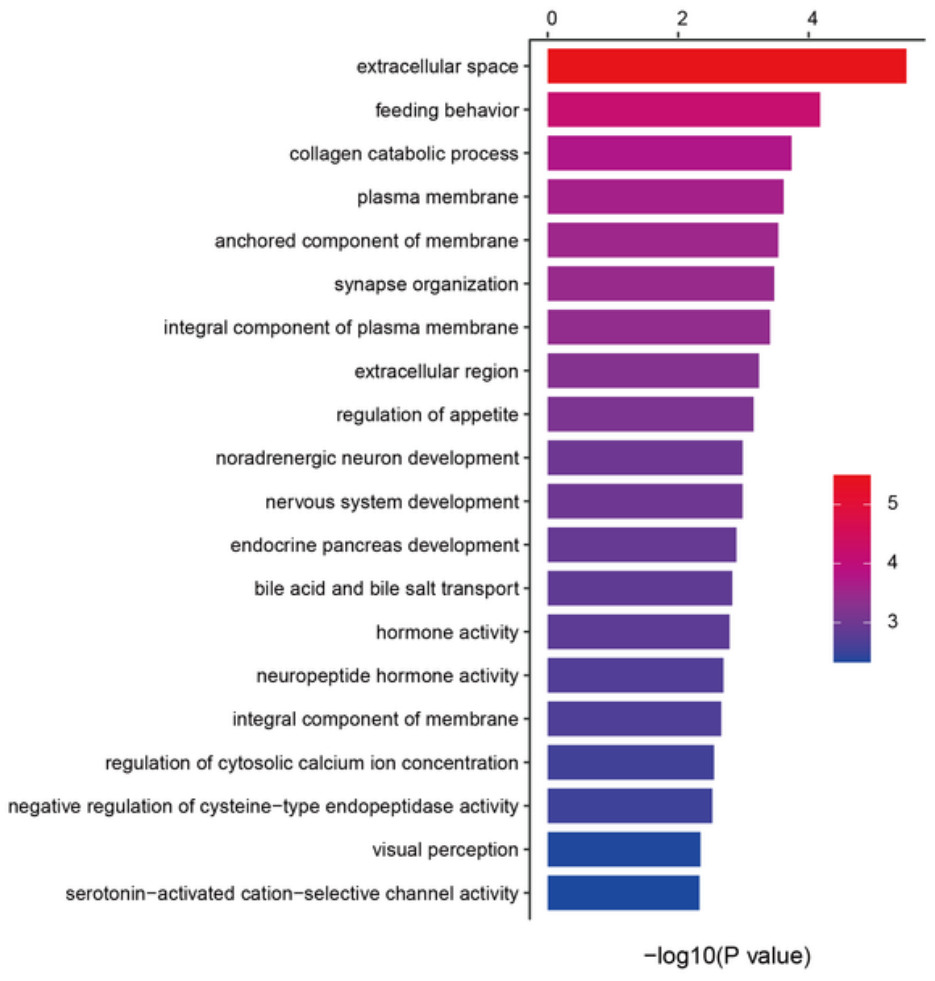

B

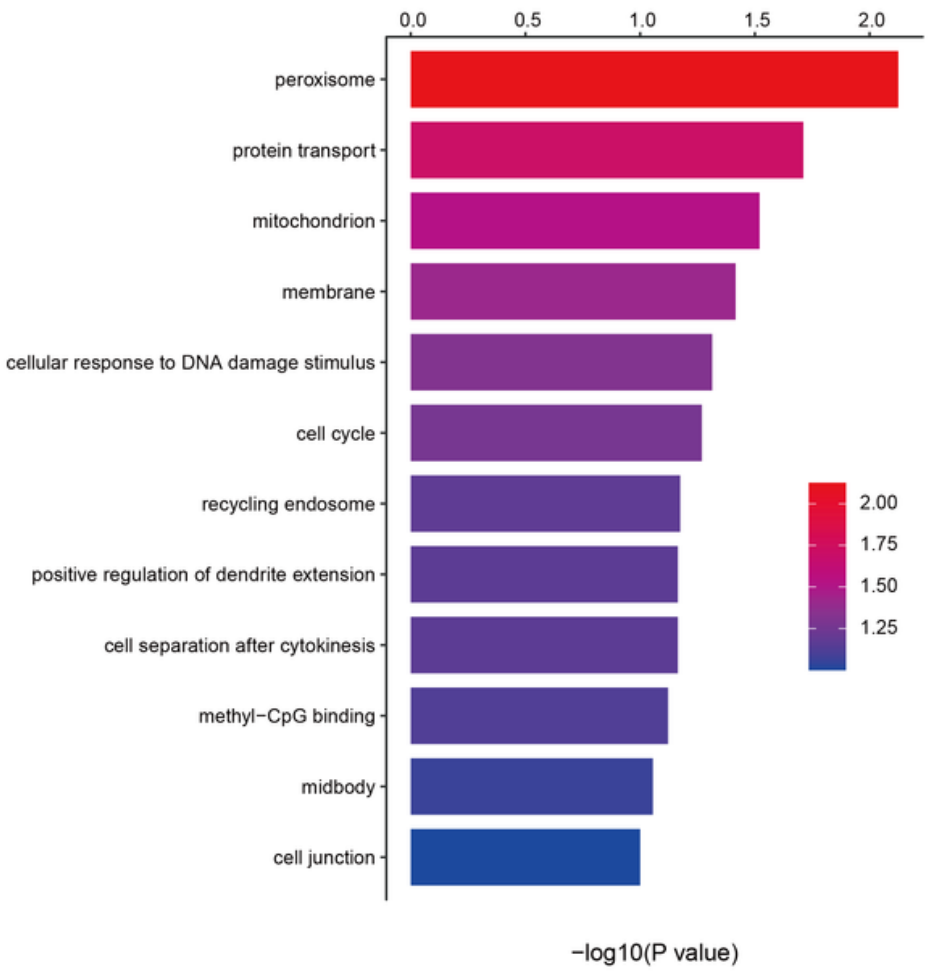

\section{Figure 3}

The top 20 enriched GO terms of SGs-LCs (A) and all GO terms enriched in SGs-LEs (B)

GO, Gene Ontology, SGs-LC, specific genes in Lynch syndrome and colorectal cancer, SGs-LEs, specific genes in Lynch syndrome and endometrial cancer 
A
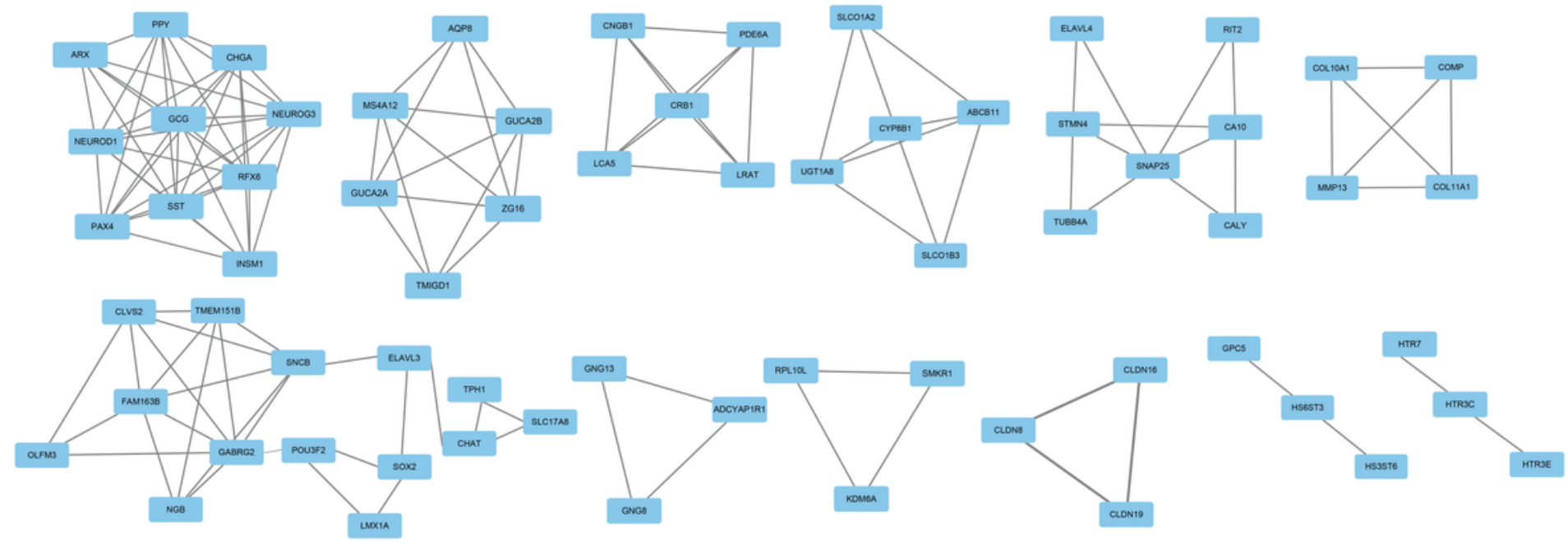

B
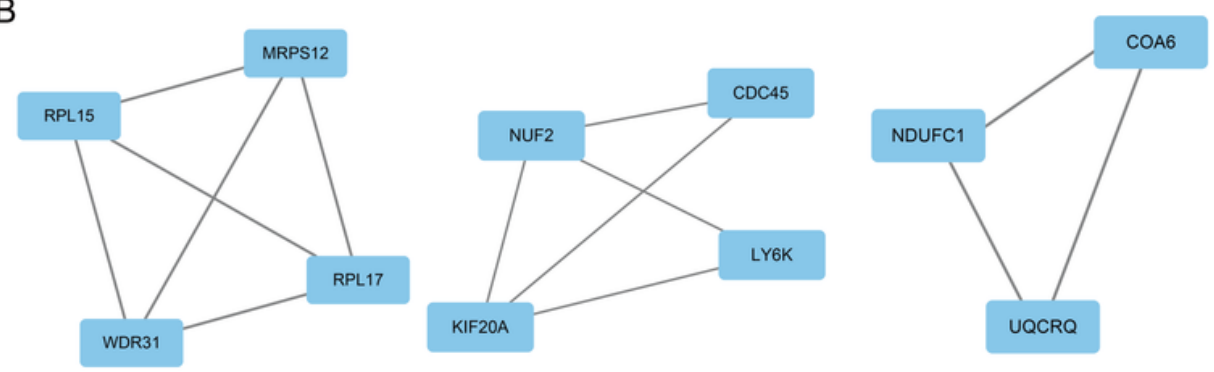

\section{Figure 4}

PPI network of SGs-LCs and SGs-LEs. SGs-LCs and SGs-LEs formed 11 clusters and 2 clusters, containing 81 and 8 genes, respectively.

PPI, protein-protein interaction, SGs-LCs, specific genes in Lynch syndrome and colorectal cancer, SGs-LEs, specific genes in Lynch syndrome and endometrial cancer 


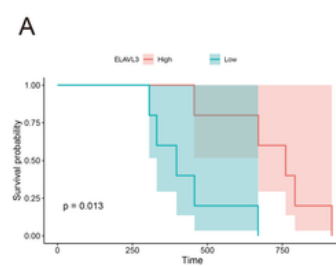

B

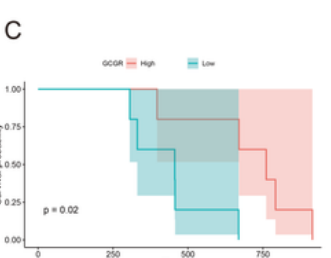

D

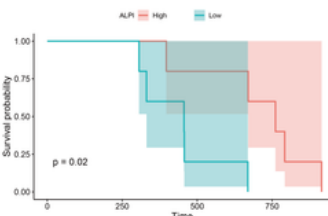

$\mathrm{E}$

$\mathrm{F}$
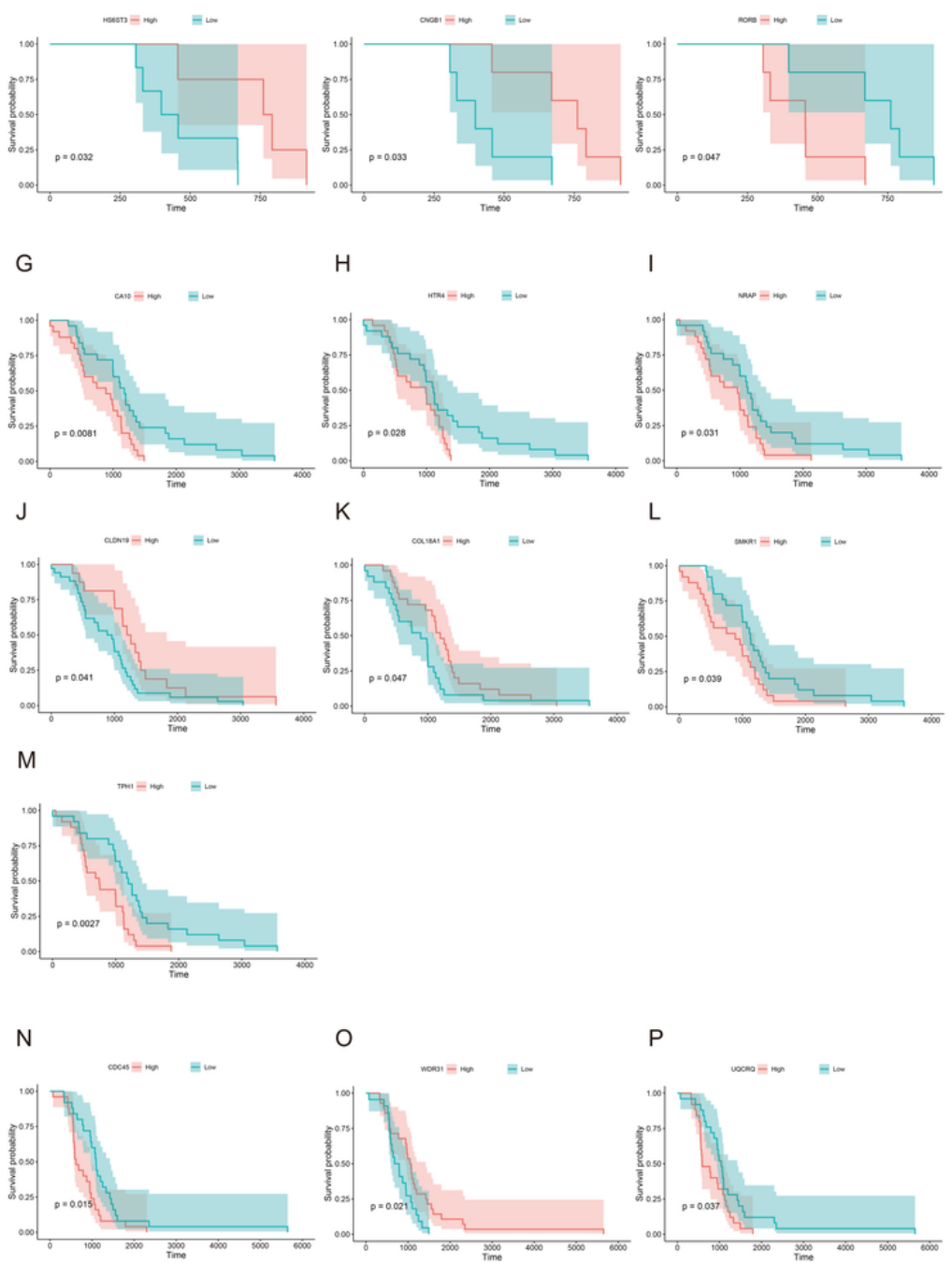

\section{Figure 5}

Genes with significant differences in survival rates between SGs-LCs and SGs-LEs at high or low expression levels.

Among the SGs-LCs, significantly different survival rates of Lynch syndrome patients were found for high vs. low expression levels of $E L A V L 3(\mathrm{~A}), A L P I(\mathrm{~B}), G C G R(\mathrm{C}), H S 6 S T 3 \mathrm{D}), C N G B 1(\mathrm{E})$ and $R O R B(\mathrm{~F})$, the survival rates of colorectal cancer patients with high vs. low expression of $C A 10(\mathrm{G}), \operatorname{HTR} 4(\mathrm{H}), \operatorname{NRAP}(\mathrm{I}), C L D N 19(\mathrm{~J})$ and COL18A1 (K) were significantly different. In EC, high and low expression levels of $C D C 45(\mathrm{~L})$ and WDR31 (M) were associated with significantly different survival rates in endometrial cancer patients.

SGs-LCs, specific genes in Lynch syndrome and colorectal cancer, SGs-LEs, specific genes in Lynch syndrome and endometrial cancer 
A
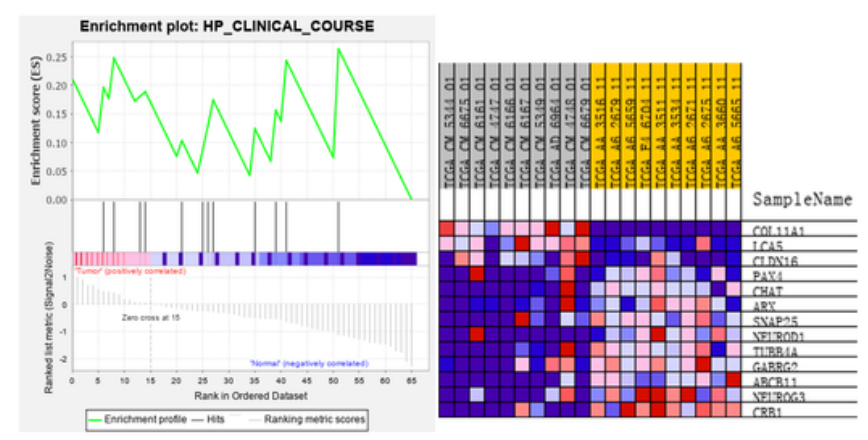

C

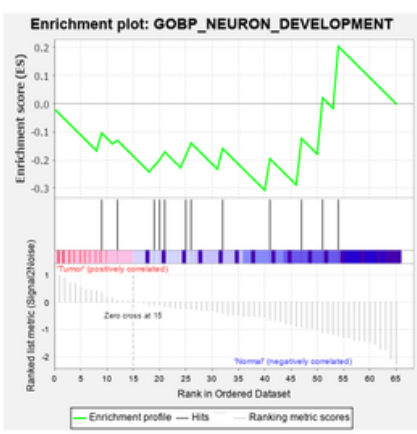

B

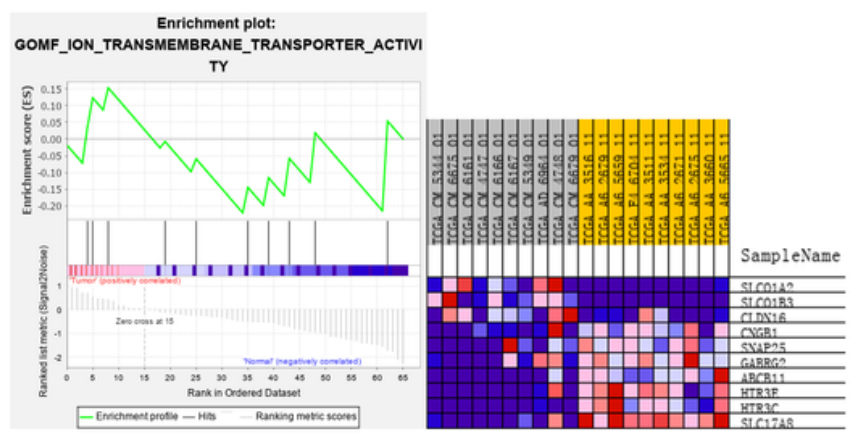

D

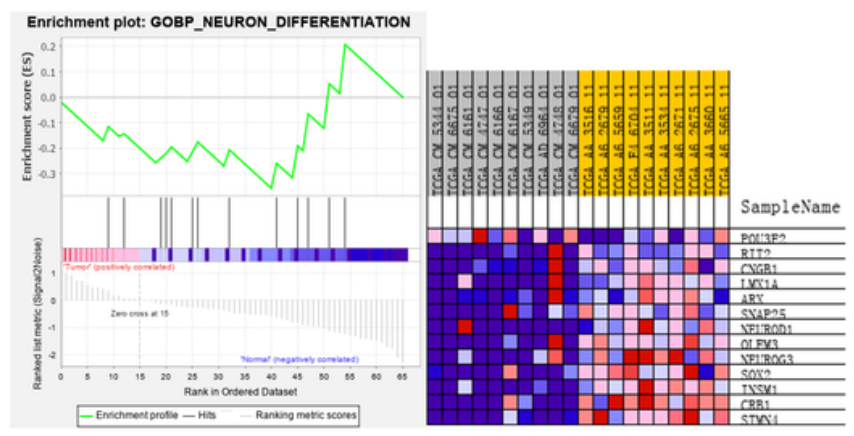

Figure 6

GSEA was carried out to identify upregulated or downregulated GO gene sets.

SGs-LCs were differentially enriched in the Lynch syndrome-related GO terms of neurogenesis $(A)$ and regulation of cell differentiation (B) and the GO terms cell_cell signalling (C), ion transport (D), neuron projection (E) and regulation of cell differentiation $(F)$ related to colorectal cancer. Red shading in the heatmap represents upregulation, and blue represents downregulation.

GSEA, Gene set enrichment analysis, GO, Gene Ontology, SGs-LCs, specific genes in Lynch syndrome and colorectal cancer

\section{Supplementary Files}

This is a list of supplementary files associated with this preprint. Click to download.

- SuppementaryTables.doc 